T. Murai

Nagoya Math. J.

Vol. 89 (1983), 65-76

\title{
THE BOUNDARY BEHAVIOUR OF HADAMARD LACUNARY SERIES
}

\author{
TAKAFUMI MURAI
}

\section{§1. Introduction}

A convergent power series $f(z)$ in the open unit disk $D$ is called Hadamard lacunary if it is expressed as follows:

$$
f(z)=\sum_{k=1}^{\infty} c_{k} z^{n_{k}}, \quad n_{k+1} / n_{k} \geq q(k \geq 1) \quad \text { for some } q>1 .
$$

We shall discuss the boundary behaviour of Hadamard lacunary series. For a subset $X$ of $D$, we put $b(X)=\bar{X} \cap \partial D$, where $\bar{X}$ is the closure of $X$ and $\partial D$ the boundary of $D$. We say that an analytic function $g(z)$ in $D$ has an extended complex number $\omega$ as an asymptotic value if there exists a path $\gamma \subset D$ with $b(\gamma) \neq \emptyset$ such that $\lim _{|z| \rightarrow 1, z \in \gamma} g(z)=\omega$. We say that $g(z)$ has an asymptotic value at $a \in \partial D$ if there exists a path $\gamma \subset D$ with $b(\gamma)=\{a\}$ such that $\lim _{z \rightarrow a, z \in r} g(z)$ exists. The Maclane class $\mathscr{A}$ is the totality of analytic functions $g(z)$ in $D$ such that $g(z)$ has asymptotic values at a dense subset of $\partial D$.

In [5], G. R. Maclane proved that a power series $f(z)$ given by (1) with $q>3$ belongs to $\mathscr{A}$. It is conjectured that Hadamard lacunary series belong to $\mathscr{A}$. In [1], J. M. Anderson noted that Maclane's result is deduced from a result of K. G. Binmore in [2]. In [3], K. G. Binmore and R. Hornblower gave an another partial answer to this question. We shall answer this question. The main purpose of this paper is to show

THEOREM. Let $f(z)$ be an Hadamard lacunary series given by (1) with $\limsup _{k \rightarrow \infty}\left|c_{k}\right|=\infty$. Then $f(z)$ has an asymptotic value $\infty$ at every point of $\partial D$.

It is known that the Hadamard lacunary series in our theorem has no finite asymptotic value ([2]), and hence $\infty$ is a unique asymptotic value.

Received April 16, 1981.

Revised October 5, 1981. 
If an Hadamard lacunary series $f(z)$ given by (1) satisfies $\limsup _{k \rightarrow \infty}\left|c_{k}\right|$ $<\infty$, then Paley's theorem ([11]) yields $f \in \mathscr{A}$. Hence we have, by our theorem,

CoRollary. Hadamard lacunary series belong to $\mathscr{A}$.

As application of our method, we shall note that Property (A) (which will be stated later) deduces Binmore's result in [2] and Sons's result on annular functions.

\section{§2. Fundamental tools}

LEMMA 1 ([4]). Let $p$ be a positive integer and $g(\zeta)$ an analytic function in $D(w, \rho)=\{\zeta ;|\zeta-w|<\rho\}$ such that $\left|g^{(p)}(w)\right| \geq y_{1}$ and $\left|g^{(p)}(\zeta)\right| \leq y_{2}$ $(\zeta \in D(w, \rho))$. Then there exists $0<\varepsilon<\rho$ such that

$$
|g(\zeta)-g(w)| \geq \eta(p) \rho^{p} y_{1}^{p+1} y_{2}^{-p}
$$

for all $\zeta \in S(w, \varepsilon)=\{z ;|z-w|=\varepsilon\}$, where $\eta(p)$ is a constant depending only on $p$.

In this lemma, we may assume that $\eta(1) \geq \eta(2) \geq \cdots$; consider $\min \{\eta(j) ; 1 \leq j \leq p\}(p=1,2, \cdots)$ if necessary.

Lemma 2 ([11]). Given $q>1$, there exist two constants $0<A \leq 1$ and $B \geq 1$ depending only on $q$ with the following property: For every lacunary polynomial $P(t)=\sum_{k=1}^{n} a_{k} e^{i m_{k} t}, m_{k+1} / m_{k} \geq q$ and every interval $I$ in $[0,2 \pi)$ of length $\geq B / m_{1}$, there exists $t_{0} \in I$ such that $\operatorname{Re} P\left(t_{0}\right) \geq A \sum_{k=1}^{n}\left|a_{k}\right|$.

Lemma 3. Let

$$
Q(\zeta)=\sum_{k=1}^{n} a_{k} \exp \left(m_{k} \zeta\right), \quad m_{k+1} / m_{k} \geq q>1(k \geq 1) .
$$

Then, for every complex number $w$ and $1 \leq d \leq n$, there exists an integer $\ell=\ell(Q, w, d)$ with $0 \leq \ell \leq n-1$ such that

$$
\left|Q^{(\ell)}(w)\right| \geq C m_{d}^{\ell}\left|a_{d}\right| \exp \left(m_{d} \operatorname{Re} w\right),
$$

where $C=1 / 2 \cdot \prod_{k=1}^{\infty}\left\{\left(1-q^{-k}\right) /\left(1+q^{-k}\right)\right\}^{2}$.

Proof. This lemma is analogous to Lemma 8 in [6]. The following elegant proof was communicated by W. H. J. Fuchs. Without loss of generality, we may assume $a_{d} \neq 0$. Let us consider an equation: 
(4)

$$
\left[\begin{array}{ccc}
1 & \cdots & 1 \\
m_{1} & \cdots & m_{n} \\
& \cdots & \cdots \\
m_{1}^{n-1} & \cdots & m_{n}^{n-1}
\end{array}\right)\left(\begin{array}{c}
x_{1} \\
\vdots \\
x_{n}
\end{array}\right)=\left(\begin{array}{c}
y_{1} \\
\vdots \\
y_{n}
\end{array}\right) .
$$

Then we have, with $\Delta=\prod_{k<j}\left(m_{j}-m_{k}\right)$,

$$
\begin{aligned}
\left|x_{d}\right| & =\left|\operatorname{det}\left(\begin{array}{ccccccc}
1 & \cdots & 1 & y_{1} & 1 & \cdots & 1 \\
\vdots & & \vdots & \vdots & \vdots & & \vdots \\
m_{1}^{n-1} & \cdots & m_{d-1}^{n-1} & y_{n} & m_{d+1}^{n-1} & \cdots & m_{n}^{n-1}
\end{array}\right)\right| / \Delta \\
& \leq \sum_{\ell=1}^{n}\left|\operatorname{det}\left(\begin{array}{c|cc}
1 & \cdots & 1 \\
\vdots & & \vdots \\
\hline \vdots & & \vdots \\
m_{1}^{n-1} & \cdots & m_{n}^{n-1}
\end{array}\right]\right|\left|y_{\ell}\right| \mid \Delta
\end{aligned}
$$

(omit the $d^{\text {th }}$ column and the $\ell^{\text {th }}$ row from the determinant in (4))

$$
=\sum_{\ell=1}^{n}\left\{\sigma_{d, \ell} \prod_{k<j ; k, j \neq d}\left(m_{j}-m_{k}\right)\right\}\left|y_{\ell}\right|\left|\Delta=\prod_{k \neq d}\right| m_{k}-\left.m_{d}\right|^{-1} \sum_{\ell=1}^{n} \sigma_{d, \ell}\left|y_{\ell}\right|,
$$

where $\sigma_{d, \ell}$ 's are defined by $\prod_{1 \leq k \leq n ; k \neq d}\left(x+m_{k}\right)=\sigma_{d, n} x^{n-1}+\sigma_{d, n-1} x^{n-2}+\cdots$ $+\sigma_{d, 1}$. If $\left|y_{\ell}\right| \leq C m_{d}^{\ell-1}\left|x_{d}\right|(\ell=1, \cdots, n)$, then

$$
\begin{aligned}
\left|x_{d}\right| & \leq \prod_{k \neq d}\left|m_{k}-m_{d}\right|^{-1} \sum_{\ell=1}^{n} \sigma_{d, \ell} m_{d}^{\ell-1} C\left|x_{d}\right| \\
& =\prod_{k \neq d}\left\{\left(m_{k}+m_{d}\right) /\left|m_{k}-m_{d}\right|\right\} C\left|x_{d}\right| \\
& \leq \prod_{k=1}^{\infty}\left\{\left(1+q^{-k}\right) /\left(1-q^{-k}\right)\right\}^{2} C\left|x_{d}\right|=\left|x_{d}\right| / 2,
\end{aligned}
$$

and hence $x_{d}=0$.

Now we put $y_{\ell}=Q^{(\ell-1)}(w)(1 \leq \ell \leq n)$ in (4). Then $x_{\ell}=a_{\ell} \exp \left(m_{\ell} w\right)$ $(1 \leq \ell \leq n)$. If (3) does not hold for all $\ell$ with $0 \leq \ell \leq n-1$, then $x_{d}=0$, that is, $a_{d}=0$. This is a contradiction. Hence (3) holds for some $\ell$ with $0 \leq \ell \leq n-1$.

\section{§3. Proof of Theorem}

In this section, we shall show that our theorem follows from two properties, which will be stated later. Let $f(z)$ be an Hadamard lacunary series given by (1) with limsup ${ }_{k \rightarrow \infty}\left|c_{k}\right|=\infty$. Our purpose is to construct a path $\gamma \subset D$ with $b(\gamma)=\{a\}$ such that $f(z)$ has $\infty$ as an asymptotic value 
along with $\gamma$. Without loss of generality, we may assume $a=1$. Adding terms with coefficient 0 if necessary, we may assume that $q \leq n_{k+1} / n_{k} \leq q^{2}$ $(k \geq 1)$.

To construct such an arc, we deal with an analytic function

$$
F(\zeta)=f\left(e^{\zeta}\right)=\sum_{k=1}^{\infty} c_{k} \exp \left(n_{k} \zeta\right)
$$

in a domain $U=\{\zeta ; \operatorname{Re} \zeta<0\}$ and shall construct a path $\Gamma \subset U$ with $b(\Gamma)$ $=\{0\}$ such that $F(\zeta)$ has $\infty$ as an asymptotic value along with $\Gamma$.

Now we introduce some notation. Throughout the paper, $A, B$ and $C$ are the constants in Lemmas 2 and 3 . We put $\theta=A / 8$. For every $-1 \leq r<0$, we put

$$
\left\{\begin{array}{cl}
M_{r}=\max \left\{\left|c_{k}\right| \exp \left(n_{k} r\right) ; k \geq 1\right\} & \text { (the maximum term) } \\
\mu_{r}=\min \left\{k ;\left|c_{k}\right| \exp \left(n_{k} r\right)=M_{r}\right\} & \text { (the smallest central index) } \\
\nu_{r}=\max \left\{k ;\left|c_{k}\right| \exp \left(n_{k} r\right)=M_{r}\right\} & \text { (the largest central index) } \\
\alpha_{r}=r-\theta / n_{\mu_{r}} \quad \text { (the smallest dominant point) } & \text { (the largest dominant point) } \\
\beta_{r}=r-\theta / n_{\nu_{r}} \quad(t t) & \\
I(t, r)=\left\{x+i t ; \alpha_{r} \leq x \leq \beta_{r}\right\} & (|t| \leq \pi) .
\end{array}\right.
$$

Then $\lim _{r \rightarrow 0} M_{r}=\lim _{r \rightarrow 0} \mu_{r}=\lim _{r \rightarrow 0} \nu_{r}=\infty$ and $\lim _{r \rightarrow 0} \alpha_{r}=\lim _{r \rightarrow 0} \beta_{r}=0$. We denote by $\left(\nu_{m}\right)_{m=1}^{\infty}\left(\nu_{m+1}>\nu_{m}\right)$ the totality of the largest central indexes. Since $\nu_{r}$ is increasing and continuous on the right, we can find $r_{m}, s_{m}$ such that $\cup\left\{r ; \nu_{r}=\nu_{m}\right\}=\left[r_{m}, s_{m}\right)(m \geq 1)$. We have $s_{m}=r_{m+1}(m \geq 1)$.

Now we prove $\mu_{s_{m}}=\nu_{m}$. Since $\mu_{r}$ is continuous on the left, we have $\mu_{s_{m}}=\lim _{r \uparrow s_{m}} \mu_{r} \leq \lim _{r \uparrow s_{m}} \nu_{r}=\nu_{m}$. Let $\mathscr{R}$ be the (finite) set of all integers with $\left|c_{k}\right| \exp \left(n_{k} s_{m}\right)=M_{s_{m}}(k \geq 1)$. Then the smallest integer in $\mathscr{R}$ is $\mu_{s_{m}}$. We have

$$
\psi_{\mu *}^{\prime}\left(s_{m}\right)<\psi_{k}^{\prime}\left(s_{m}\right) \quad\left(k \in \mathscr{R}, k \neq \mu^{*}\right),
$$

where $\mu^{*}=\mu_{s_{m}}$ and $\psi_{k}(r)=\left|c_{k}\right| \exp \left(n_{k} r\right)$. Hence $\left|c_{\mu^{*}}\right| \exp \left(n_{\mu^{*}} r\right)>\left|c_{k}\right| \exp \left(n_{k} r\right)$ $\left(\mu^{*}<k \leq \nu_{m+1}\right)$ for all $r\left(r<s_{m}\right)$ sufficiently near to $s_{m}$. Since $\nu_{r} \leq \nu_{m+1}$ $\left(r \leq s_{m}\right)$, this signifies $\nu_{r} \leq \mu_{s_{m}}$ for all $r\left(r<s_{m}\right)$ sufficiently near to $s_{m}$. Thus $\nu_{m}=\lim _{r \uparrow s_{m}} \nu_{r} \leq \mu_{s_{m}} \leq \nu_{m}$. Consequently, $\mu_{s_{m}}=\mu_{r_{m+1}}=\nu_{m}$. By these facts, we have $\bigcup\left\{\beta_{r} ;-1 \leq r<0, \nu_{r}=\nu_{m}\right\}=\left[\beta_{r_{m}}, \alpha_{r_{m+1}}\right)(m \geq 1)$.

For every $-1 \leq r<0$, we denote by $\xi_{r}$ the largest integer in a set of $m$ 's $(m \geq 1)$ with $\sum_{k<m}\left|c_{k}\right| \leq A / 2 \cdot M_{r}$; if the set is empty, we put $\xi_{r}=0$. Then $\lim _{r \rightarrow 0} \xi_{r}=\infty$. We need the following two properties. 
(A) For every $w$ with $\beta_{r_{m}} \leq \operatorname{Re} w \leq \alpha_{r_{m+1}}$ (for some $m$ ), there exists a positive number $\varepsilon_{w}$ with $0<\varepsilon_{w} \leq 1 / n_{\nu_{m}}$ such that $|F(\zeta)| \geq D M_{r_{m}}\left(\zeta \in S\left(w, \varepsilon_{w}\right)\right)$, where $D$ is a constant depending only on $q$.

(B) For every $m \geq 2$, there exist a point $t_{m}$ with $\left|t_{m}\right| \leq 2 B / n_{\xi}\left(\xi=\xi_{r_{m}}\right)$ and a corresponding Jordan curve $\Gamma_{m}$ with diam $\left(\Gamma_{m}\right)=$ (the diameter of $\left.\Gamma_{m}\right) \leq 3 / n_{\nu_{m-1}}$ such that $\left\langle\Gamma_{m}\right\rangle \supset I\left(t_{m}, r_{m}\right)$ and $|F(\zeta)| \geq E M_{r_{m}}\left(\zeta \in \Gamma_{m}\right)$, where $\left\langle\Gamma_{m}\right\rangle$ is the domain bounded by $\Gamma_{m}$ and $E$ a constant depending only on $q$.

We postpone the proof of (A) and (B) to the sections 4 and 5. From now, we construct a required path $\Gamma$ assuming (A) and (B).

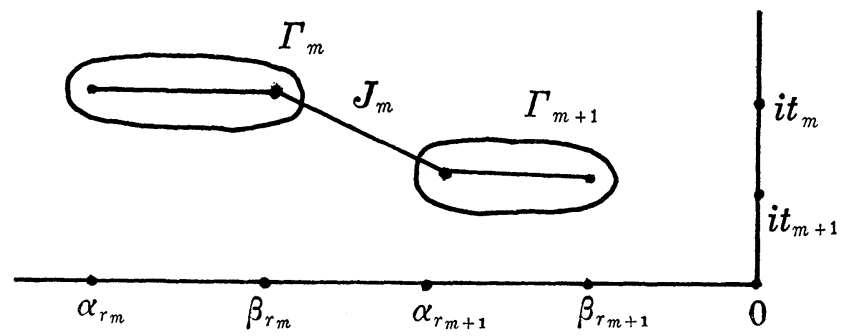

Fig.

Note that $\left[\beta_{r_{1}}, 0\right)=\bigcup_{m=1}^{\infty}\left[\beta_{r_{m}}, \alpha_{r_{m+1}}\right] \cup\left[\alpha_{r_{m+1}}, \beta_{r_{m+1}}\right]$. Let $J_{m}$ be the segment which connects $\beta_{r_{m}}+i t_{m}$ and $\alpha_{r_{m+1}}+i t_{m+1}(m \geq 1)$. The property (A) shows that, for every $w \in J_{m}$, there exists $0<\varepsilon_{w} \leq 1 / n_{\nu_{m}}$ such that $|F(\zeta)|$ $\geq D M_{r_{m}}\left(\zeta \in S\left(w, \varepsilon_{w}\right)\right)$. This shows that there exists a Jordan curve $\gamma_{m}$ with $\kappa_{m}=\max$ the distance of $\zeta$ and $\left.J_{m} ; \zeta \in \gamma_{m}\right\} \leq 1 / n_{\nu_{m}}$ such that $\left\langle\gamma_{m}\right\rangle \supset$ $J_{m}$ and $|F(\zeta)| \geq D M_{r_{m}}\left(\zeta \in \gamma_{m}\right)$. Put $\Gamma^{*}=\bigcup_{m=1}^{\infty}\left(\Gamma_{m} \cup \gamma_{m}\right)$. Since $\lim _{m \rightarrow \infty} t_{m}$ $=0$, we have $b\left(\Gamma^{*}\right) \ni 0$. Since $\sum_{j=m}^{\infty} \operatorname{diam}\left(\Gamma_{j}\right)+\sum_{j=m}^{\infty} \kappa_{j}=o(1)(m \rightarrow \infty)$, we have $b\left(\Gamma^{*}\right)=\{0\}$. Since $\Gamma^{*}$ is arcwise connected, we can choose a path $\Gamma \subset \Gamma^{*}$ with $b(\Gamma)=\{0\}$. Then $F(\zeta)$ has $\infty$ as an asymptotic value along with $\Gamma$.

\section{§4. Proof of (A)}

In this section, we prove (A). Let $w$ satisfy $\beta_{r_{m}} \leq \operatorname{Re} w \leq \alpha_{r_{m+1}}$. Put $r=\operatorname{Re} w+\theta / n_{\nu_{m}}$. Then $r_{m} \leq r \leq r_{m+1}$ and $M_{r}=\left|c_{\nu_{m}}\right| \exp \left(n_{\nu_{m}} r\right)$.

Lemma 4. There exists a positive integer $p=p(F, w)$ with $1 \leq p \leq N$ ( $N$ : a constant depending only on $q$ ) such that

$$
\left|F^{(p)}(w)\right| \geq C / 2 e \cdot M_{r} n_{\nu_{m}}^{p} .
$$


Proof. Let us write

$$
\begin{aligned}
F^{\prime}(\zeta) & =\sum_{k=1}^{\infty} n_{k} c_{k} \exp \left(n_{k} \zeta\right)=\sum_{k<\nu_{m}-n}+\sum_{\nu_{m}-n \leq k \leq \nu_{m}+n}+\sum_{k>\nu_{m}+n} \\
& =\phi(\zeta)+Q(\zeta)+\Phi(\zeta),
\end{aligned}
$$

where $n$ is determined later. Lemma 3 shows that there exists $\ell=\ell(n)$ with $0 \leq \ell \leq 2 n$ such that

$$
\left|Q^{(\ell)}(w)\right| \geq C n_{\nu_{m}}^{\ell+1}\left|c_{\nu_{m}}\right| \exp \left\{n_{\nu_{m}}\left(r-\theta / n_{\nu_{m}}\right)\right\} \geq C / e \cdot M_{r} n_{\nu_{m}}^{\ell+1} .
$$

We have

$$
\begin{aligned}
\left|\phi^{(\ell)}(w)\right| & \leq M_{r} \sum_{k<\nu_{m}-n} n_{k}^{\ell+1}=M_{r} n_{\nu_{m}}^{\ell+1} \sum_{k<\nu_{m}-n}\left(n_{k} / n_{\nu_{m}}\right)^{\ell+1} \\
& \leq M_{r} n_{\nu_{m}}^{\ell+1} \sum_{j=n+1}^{\infty} q^{-j(\ell+1)} \leq\left\{1 / q^{n}(q-1)\right\} M_{r} n_{\nu_{m}}^{\ell+1} .
\end{aligned}
$$

Note that $x^{2 n+1} e^{-\theta x}$ is decreasing in $[(2 n+1) / \theta, \infty)$. We choose an integer $N_{0}=N_{0}(\theta)$ so that $q^{j} \geq(2 j+1) / \theta\left(j \geq N_{0}(\theta)\right)$. Let $n \geq N_{0}$. Then

$$
\begin{aligned}
\left|\Phi^{(\ell)}(w)\right| & \leq \sum_{k>\nu_{m}+n} n_{k}^{\ell+1}\left|c_{k}\right| \exp \left\{n_{k}\left(r-\theta / n_{\nu_{m}}\right)\right\} \\
& \leq M_{r} \sum_{k>\nu_{m}+n} n_{k}^{\ell+1} \exp \left(-\theta n_{k} / n_{\nu_{m}}\right) \\
& \leq M_{r} n_{\nu_{m}}^{\ell+1} \sum_{k>\nu_{m}+n}\left(n_{k} / n_{\nu_{m}}\right)^{2 n+1} \exp \left(-\theta n_{k} / n_{\nu_{m}}\right) \\
& \leq M_{r} n_{\nu_{m}}^{\ell+1} \sum_{j=n+1}^{\infty} q^{j(2 n+1)} \exp \left(-\theta q^{j}\right)\left(=M_{r} n_{\nu_{m}}^{\ell+1} \tau_{n}(\theta), \text { say }\right) .
\end{aligned}
$$

Now we choose $n\left(\geq N_{0}\right)$ so that $1 / q^{n}(q-1) \leq C / 4 e, \tau_{n}(\theta) \leq C / 4 e$ and put $p=p(F, w)=\ell(n)+1, N=2 n+1$. Then (8) follows from (9), (10) and (11).

Q.E.D.

Lemma 5. Let $p=p(F, w)$ be the integer in Lemma 4. Then, for any $\zeta \in D\left(w, 1 / 2 n_{\nu_{m}}\right)$,

$$
\left|F^{(p)}(\zeta)\right| \leq D_{0} M_{r} n_{\nu_{m}}^{p}
$$

where $D_{0}=\left\{1+(2 / \theta)^{2 N}(2 N) !\right\} q /(q-1)$.

Proof. Note that $e^{-\theta x / 2} \leq(2 / \theta)^{2 p}(2 p) ! x^{-2 p}(x>0)$. Since $\operatorname{Re} \zeta \leq r-$ $\theta / 2 n_{\nu_{m}}$, we have

$$
\begin{aligned}
\left|F^{(p)}(\zeta)\right| & \leq \sum_{k=1}^{\infty} n_{k}^{p}\left|c_{k}\right| \exp \left\{n_{k}\left(r-\theta / 2 n_{\nu_{m}}\right)\right\} \\
& \leq M_{r} \sum_{k=1}^{\infty} n_{k}^{p} \exp \left(-\theta n_{k} / 2 n_{\nu_{m}}\right)=M_{r}\left\{\sum_{k=1}^{\nu_{m}}+\sum_{k=\nu_{m}+1}^{\infty}\right\}
\end{aligned}
$$




$$
\begin{aligned}
& \leq M_{r} n_{\nu_{m}}^{p}\left\{\sum_{k=1}^{\nu_{m}}\left(n_{k} / n_{\nu_{m}}\right)^{p}+(2 / \theta)^{2 p}(2 p) ! \sum_{k=\nu_{m}+1}^{\infty}\left(n_{k} / n_{\nu_{m}}\right)^{p}\left(n_{\nu_{m}} / n_{k}\right)^{2 p}\right\} \\
& \leq M_{r} n_{\nu_{m}}^{p}\left\{1+(2 / \theta)^{2 p}(2 p) !\right\} \sum_{j=0}^{\infty} q^{-p j} \leq D_{0} M_{r} n_{\nu_{m}}^{p} .
\end{aligned}
$$

Now we apply Lemma 1 to $g(\zeta)=F(\zeta)$ and $D\left(w, \theta / 2 n_{\nu_{m}}\right)$. There exists $0<\varepsilon \leq \theta / 2 n_{\nu_{m}}\left(\leq 1 / n_{\nu_{m}}\right)$ such that, for any $\zeta \in S(w, \varepsilon)$,

$$
\begin{aligned}
\mid F(\zeta) & -F(w) \mid \geq \eta(p)\left(\theta / 2 n_{\nu_{m}}\right)^{p}\left(C / 2 e \cdot M_{r} n_{\nu_{m}}^{p}\right)^{p+1}\left(D_{0} M_{r} n_{\nu_{m}}^{p}\right)^{-p} \\
& =\left\{\eta(p)(\theta / 2)^{p}(C / 2 e)^{p+1} D_{0}^{-p}\right\} M_{r} \\
& \geq\left\{\eta(N)(\theta / 2)^{N}(C / 2 e)^{N+1} D_{0}^{-N}\right\} M_{r_{m}}\left(=3 D M_{r_{m}}, \text { say }\right) .
\end{aligned}
$$

If $|F(w)|<2 D M_{r_{m}}$, then $|F(\zeta)| \geq D M_{r_{m}}(\zeta \in S(w, \varepsilon))$. Hence $\varepsilon_{w}=\varepsilon$ is a required number. If $|F(w)| \geq 2 D M_{r_{m}}$, we choose $0<\varepsilon_{w} \leq 1 / n_{\nu_{m}}$ so that $|F(\zeta)| \geq D M_{r_{m}}\left(\zeta \in S\left(w, \varepsilon_{w}\right)\right)$. This completes the proof of (A).

\section{§5. Proof of (B)}

In this section, we prove (B). For the sake of simplicity, we write, for a polynomial $P(t)=\sum_{k=1}^{n} a_{k} e^{i m_{k} t},\|P\|=\sum_{k=1}^{n}\left|a_{k}\right|, \ell(P)=$ (the length of $P)=n$, s.e. $P=($ the smallest exponent in $P)=m_{1}$, l.e. $P=($ the largest exponent in $P$ ) $=m_{n}$.

Given $m \geq 2$, our purpose is to define a point $t_{m}$ and a corresponding Jordan curve $\Gamma_{m}$ having the required properties. We write simply $r=r_{m}$, $\xi=\xi_{m}, \mu=\mu_{r_{m}}\left(=\nu_{m-1}\right), \nu=\nu_{m}$. We need two constants $\lambda, \Lambda$ depending only on $q$ which are defined as follows.

Let $\lambda$ be a positive integer such that $B /\left\{\theta q^{\lambda-1}(q-1)\right\} \leq A / 32$ and $\Lambda$ a positive integer such that $(A / 2+1) / \Lambda \leq A / 4$.

Using $\lambda, \Lambda$, we define polynomials $\bar{\Delta}_{0}, \underline{\Delta}_{1}, \bar{\Delta}_{1}, \underline{\Delta}_{2}, \bar{\Delta}_{2}, \ldots$ with $\ell\left(\bar{\Delta}_{j}\right) \leq$ $2 \lambda(\Lambda-1), \quad \ell\left(\underline{\Delta}_{j}\right)=\lambda(j \geq 1) . \quad$ Let $\Delta_{0}^{*}(t)=\sum_{k=\xi}^{\mu} c_{k} \exp \left\{n_{k}(r+i t)\right\}, \quad \Delta_{\ell}^{*}(t)=$ $\sum_{\mu+\lambda(\ell-1)<k \leq \mu+\lambda \ell} c_{k} \exp \left\{n_{k}(r+i t)\right\} \quad(\ell \geq 1)$. Choosing a sequence $\left(\ell_{j}\right)_{j=1}^{\infty}$ of positive integers so that $\left\|\Delta_{\ell_{j}}^{*}\right\|=\min \left\{\left\|\Delta_{\ell}^{*}\right\| ; \Lambda(j-1)<\ell \leq \Lambda j\right\}$, we put $\bar{\Delta}_{0}$

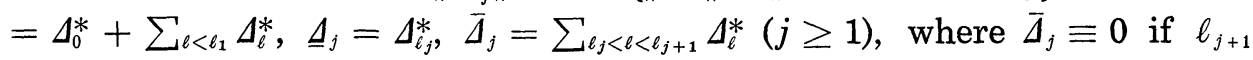
$=\ell_{j}+1$. Thus the required polynomials are defined. We put $h_{j}=$ s.e. $\bar{\Delta}_{j}$ $(j \geq 1), H_{j}=1$.e. $\bar{\Delta}_{j}(j \geq 0)$, where $h_{j}=H_{j}=1$. e. $\underline{\Delta}_{j}$ if $\bar{\Delta}_{j} \equiv 0$. Denoting by $\sigma$ the smallest non-negative integer such that $n_{\nu} \leq H_{j}(j \geq 0)$, we put $S_{0}=\bar{\Delta}_{0}, S_{j}=\sum_{\ell=0}^{j} \bar{\Delta}_{\ell}+\sum_{\ell=1}^{j} \underline{\Delta}_{\ell}(1 \leq j \leq \sigma)$. Then s.e. $S_{0}=n_{\xi}$, l.e. $S_{j}=H_{j}$ $(0 \leq j \leq \sigma)$. The required point $t_{m}$ is defined by

Lemma 6. There exists $t_{m}$ with $\left|t_{m}\right| \leq 2 B / n_{\xi}$ such that $\left|S_{j}\left(t_{m}\right)\right| \geq A / 4 \cdot\left\|S_{j}\right\|$ $(0 \leq j \leq \sigma)$. 
Proof. Using Lemma 2, we define inductively $\sigma+1$ points $\left(u_{j}\right)_{j=0}^{\sigma}$ in the following manner: Let $u_{0}$ be a point with $\left|u_{0}\right| \leq B / n_{\xi}$ such that $\operatorname{Re} S_{0}\left(u_{0}\right)$ $\geq A\left\|S_{0}\right\|$ and $u_{j}$ a point with $\left|u_{j}-u_{j-1}\right| \leq B / h_{j}$ such that $\operatorname{Re} \bar{\Delta}_{j}\left(u_{j}\right) \geq A\left\|\bar{\Delta}_{j}\right\|$ $(1 \leq j \leq \sigma)$. We put $t_{m}=u_{\sigma}$ and prove that this is a required point.

We have

$$
\begin{aligned}
\mid u_{j}- & t_{m} \mid \leq B \sum_{\ell>j} 1 / h_{\ell} \\
& =B / H_{j} \sum_{\ell>j}\left(H_{j} / h_{\ell}\right) \leq B /\left\{q^{2-1}(q-1) H_{j}\right\} \leq A /\left(2 H_{j}\right) \quad(0 \leq j \leq \sigma) .
\end{aligned}
$$

In particular, $\left|t_{m}\right| \leq\left|u_{0}\right|+A /\left(2 H_{0}\right) \leq B / n_{\xi}+A /\left(2 n_{\xi}\right) \leq 2 B / n_{\xi}$. By (14), we have

$$
\begin{aligned}
& \operatorname{Re} \bar{\Delta}_{j}\left(t_{m}\right) \geq \operatorname{Re} \bar{\Delta}_{j}\left(u_{j}\right)-\left|u_{j}-t_{m}\right|\left\|\bar{\Delta}_{j}^{\prime}\right\| \\
& \quad \geq A\left\|\bar{\Delta}_{j}\right\|-\left(A / 2 H_{j}\right) H_{j}\left\|\bar{\Delta}_{j}\right\| \geq A / 2 \cdot\left\|\bar{\Delta}_{j}\right\| \quad(1 \leq j \leq \sigma)
\end{aligned}
$$

and $\operatorname{Re} S_{0}\left(t_{m}\right) \geq \operatorname{Re} S_{0}\left(u_{0}\right)-\left|u_{0}-t_{m}\right| H_{0}\left\|S_{0}\right\| \geq A / 2 \cdot\left\|S_{0}\right\|$. Hence the required inequality holds for $j=0$. Let $1 \leq j \leq \sigma$. Then (15) gives

$$
\begin{aligned}
\left|S_{j}\left(t_{m}\right)\right| & \geq \operatorname{Re} S_{j}\left(t_{m}\right) \geq \operatorname{Re} S_{0}\left(t_{m}\right)+\sum_{\ell=1}^{j} \operatorname{Re} \bar{\Delta}_{\ell}\left(t_{m}\right)-\sum_{\ell=1}^{j}\left\|\underline{\Delta}_{\ell}\right\| \\
& \geq A / 2 \cdot\left(\left\|S_{0}\right\|+\sum_{\ell=1}^{j}\left\|\bar{\Delta}_{\ell}\right\|\right)-\sum_{\ell=1}^{j}\left\|\underline{\Delta}_{\ell}\right\| \\
& \geq\{A / 2 \cdot(1-1 / \Lambda)-1 / \Lambda\}\left\|S_{j}\right\| \geq A / 4 \cdot\left\|S_{j}\right\| .
\end{aligned}
$$

To define the required Jordan curve $\Gamma_{m}$, we assume, for a while, $\sigma \geq 2$ and consider intervals $\left[r-\theta / n_{\mu}, r-\theta / H_{0}\right],\left[r-\theta / H_{j-1}, r-\theta / H_{j}\right]$ $(1 \leq j \leq \sigma-1),\left[r-\theta / H_{\sigma-1}, r-\theta / n_{\nu}\right]$. We prepare

LEMma 7. If $x \in\left[r-\theta / H_{j-1}, r-\theta / H_{j}\right]$ for some $1 \leq j \leq \sigma-1$, then

$$
\left|F\left(x+i t_{m}\right)\right| \geq A / 16 \cdot M_{r}-T_{j},
$$

where $T_{j}=\left\|\underline{\Delta}_{j}\right\|+\left\|\bar{\Delta}_{j}\right\|+\left\|\underline{\Delta}_{j+1}\right\|$.

Proof. Writing

$$
\begin{aligned}
F\left(x+i t_{m}\right) & =\sum_{k=1}^{\infty} c_{k} \exp \left\{n_{k}\left(x+i t_{m}\right)\right\} \\
& =\sum_{k<\xi}+\sum_{n_{\xi} \leq n_{k} \leq H_{j-1}}+\sum_{H_{j-1}<n_{k}<h_{j+1}}+\sum_{n_{k} \geq h_{j+1}},
\end{aligned}
$$

we denote by $S_{j-1, x}$ the second term. Then

$$
\begin{aligned}
\left|S_{j-1, x}\right| & \geq\left|S_{j-1}\left(t_{m}\right)\right|-|x-r|\left\|S_{j-1}^{\prime}\right\| \\
& \geq A / 4 \cdot\left\|S_{j-1}\right\|-\left(\theta / H_{j-1}\right) H_{j-1}\left\|S_{j-1}\right\|=A / 8 \cdot\left\|S_{j-1}\right\| \geq A / 8 \cdot M_{r} .
\end{aligned}
$$


On the other hand, the sum of absolute values of other terms is dominated by

$$
\begin{aligned}
& \sum_{k<\xi}\left|c_{k}\right|+\left\{\left\|\underline{\Delta}_{j}\right\|+\left\|\bar{\Delta}_{j}\right\|+\left\|\underline{\Delta}_{j+1}\right\|\right\}+M_{r} \sum_{n_{k} \sum_{j+1}} \exp \left(-\theta n_{k} / H_{j}\right) \\
& \leq A / 32 \cdot M_{r}+T_{j}+1 /\left\{\theta q^{\alpha-1}(q-1)\right\} \cdot M_{r} \leq A / 16 \cdot M_{r}+T_{j} .
\end{aligned}
$$

Hence we have (16).

Q.E.D.

For the definition of $\Gamma_{m}$, we choose, for every $x \in\left[\alpha_{r}, \beta_{r}\right]$, a number $0<\varepsilon_{x} \leq 1 / n_{\mu}$ such that $|F(\zeta)| \geq E M_{r}\left(\zeta \in S\left(x+i t_{m}, \varepsilon_{x}\right)\right)$ (E: some constant). Let $x \in\left[r-\theta / H_{j-1}, r-\theta / H_{j}\right] \quad(1 \leq j \leq \sigma-1)$. We must distinguish the following two cases:
(a) $T_{j}<A / 32 \cdot M_{r}$,
(b) $T_{\mathrm{\jmath}} \geq A / 32 \cdot M_{r}$.

In the case (a), we have $\left|F\left(x+i t_{m}\right)\right| \geq A / 32 \cdot M_{r}$ and hence we can choose $0<\varepsilon_{x} \leq 1 / n_{\mu}$ so that $|F(\zeta)| \geq E_{1} M_{r}\left(\zeta \in S\left(x+i t_{m}, \varepsilon_{x}\right)\right)$ with $E_{1}=A / 64$. In the case (b), the choice of $\varepsilon_{x}$ will be analogous as in the proof of (A).

Since $T_{j} \geq A / 32 \cdot M_{r}, \ell\left(\underline{\Delta}_{j}+\bar{\Delta}_{j}+\underline{\Delta}_{j+1}\right) \leq 2 \lambda \Lambda$, there exists $d$ with $H_{j-1}$ $<n_{d} \leq h_{j+1}$ such that $\left|c_{d}\right| \exp \left(n_{d} r\right) \geq A /(64 \lambda \Lambda) \cdot M_{r}$. Hence $\left|c_{d}\right| \exp \left(n_{d} x\right) \geq$ $\left|c_{d}\right| \exp \left(n_{d} r-\theta n_{d} / H_{j-1}\right) \geq A /\left\{64 \lambda \Lambda \exp \left(\theta q^{4 \lambda A}\right)\right\} \cdot M_{r}\left(=3 E_{2} M_{r}\right.$, say). First we prove that there exists a positive integer $p^{\prime}=p^{\prime}(F, x)$ with $1 \leq p^{\prime} \leq N^{\prime}$ $\left(N^{\prime}\right.$ : a constant depending only on $q$ ) such that

$$
\left|F^{\left(p^{\prime}\right)}\left(x+i t_{m}\right)\right| \geq C E_{2} M_{r} n_{d}^{p^{\prime}} .
$$

Let us write

$$
\begin{aligned}
F^{\prime}(\zeta) & =\sum_{k=1}^{\infty} n_{k} c_{k} \exp \left(n_{k} \zeta\right)=\sum_{k<d-n^{\prime}}+\sum_{d-n^{\prime} \leq k \leq d+n^{\prime}}+\sum_{k>d+n^{\prime}} \\
& =\phi(\zeta)+Q(\zeta)+\Phi(\zeta),
\end{aligned}
$$

where $n^{\prime}$ will be determined later; we choose, for a while, so that $n^{\prime} \geq$ $N_{0}^{\prime}\left(N_{0}^{\prime}=N_{0}\left(\theta q^{-4 \lambda \lambda}\right)\right.$ : the function given in the proof of Lemma 4). Lemma 3 shows that there exists $\ell=\ell\left(n^{\prime}\right)$ with $0 \leq \ell \leq 2 n^{\prime}$ such that

$$
\left|Q^{(\ell)}\left(x+i t_{m}\right)\right| \geq 3 C E_{2} M_{r} n_{d}^{\ell+1}
$$

We have

$$
\left|\phi^{(\ell)}\left(x+i t_{m}\right)\right| \leq\left\{1 / q^{n^{\prime}}(q-1)\right\} M_{r} n_{d}^{\ell+1} \quad(\text {; see }(10)) .
$$

Since $x \leq r-\theta / H_{j}=r-\left(\theta n_{d} / H_{j}\right) / n_{d} \leq r-\left(\theta q^{-4 \lambda A}\right) / n_{d}$, we have 


$$
\begin{aligned}
& \left|\Phi^{(\ell)}\left(x+i t_{m}\right)\right| \leq M_{r} \sum_{k>d+n^{\prime}} n_{k}^{\ell+1} \exp \left(-\theta n_{k} / H_{j}\right) \\
& \quad \leq M_{r} n_{d}^{\ell+1} \sum_{k>d+n^{\prime}}\left(n_{k} / n_{d}\right)^{2 n^{\prime}+1} \exp \left\{-\left(\theta q^{-4 \lambda A}\right)\left(n_{k} / n_{d}\right)\right\} \\
& \leq M_{r} n_{d}^{\ell+1} \tau_{n^{\prime}}\left(\theta q^{-4 \lambda A}\right) \quad(; \text { see }(11)) .
\end{aligned}
$$

Choosing $n^{\prime}\left(\geq N_{0}^{\prime}\right)$ so that $1 / q^{n^{\prime}}(q-1) \leq C E_{2}, \tau_{n^{\prime}}\left(\theta q^{-4 \lambda \lambda}\right) \leq C E_{2}$, we put $p^{\prime}=p^{\prime}(F, x)=\ell\left(n^{\prime}\right)+1, N^{\prime}=2 n^{\prime}+1$. Then (17) follows from (18), (19) and (20).

Next we prove

$$
\left|F^{\left(p^{\prime}\right)}(\zeta)\right| \leq E_{3} M_{r} n_{d}^{p^{\prime}} \quad\left(\zeta \in D\left(x+i t_{m}, \theta / 2 H_{j}\right)\right),
$$

where $E_{3}=\left\{1+(2 / \theta)^{2 N^{\prime}}\left(2 N^{\prime}\right) ! q^{4 N^{\prime} \lambda \lambda}\right\} q /(q-1)$.

Since $\operatorname{Re} \zeta \leq x+\theta / 2 H_{j} \leq r-\theta / 2 H_{j}$, we have

$$
\begin{aligned}
\left|F^{\left(p^{\prime}\right)}(\zeta)\right| & \leq M_{r} \sum_{k=1}^{\infty} n_{k}^{p^{\prime}} \exp \left(-\theta n_{k} / 2 H_{j}\right)=M_{r}\left\{\sum_{k=1}^{d}+\sum_{k=d+1}^{\infty}\right\} \\
& \leq M_{r} n_{d}^{p^{\prime}}\left\{\sum_{k=1}^{d}\left(n_{k} / n_{d}\right)^{p^{\prime}}+(2 / \theta)^{2 p^{\prime}}\left(2 p^{\prime}\right) ! \sum_{k=d+1}^{\infty}\left(n_{k} / n_{d}\right)^{p^{\prime}}\left(H_{j} / n_{k}\right)^{2 p^{\prime}}\right\} \\
& \leq M_{r} n_{d}^{p^{\prime}}\left\{q^{p^{\prime}} /\left(q^{p^{\prime}}-1\right)+(2 / \theta)^{2 p^{\prime}}\left(2 p^{\prime}\right) !\left(H_{j} / n_{d}\right)^{2 p^{\prime}} \sum_{k=d+1}^{\infty}\left(n_{d} / n_{k}\right)^{p^{\prime}}\right\} \\
& \leq M_{r} n_{d}^{p^{\prime}}\left\{1+(2 / \theta)^{2 p^{\prime}}\left(2 p^{\prime}\right) ! q^{4 p^{\prime} \lambda \lambda}\right\} q^{p^{\prime}} /\left(q^{p^{\prime}}-1\right) \leq E_{3} M_{r} n_{d}^{p^{\prime}} .
\end{aligned}
$$

Now we apply Lemma 1 to $g(\zeta)=F(\zeta)$ and $D\left(x+i t_{m}, \theta / 2 H_{j}\right)$. There exists $0<\varepsilon \leq \theta / 2 H_{j}$ such that, for any $\zeta \in S\left(x+i t_{m}, \varepsilon\right)$,

$$
\begin{aligned}
& \left|F(\zeta)-F\left(x+i t_{m}\right)\right| \geq \eta\left(p^{\prime}\right)\left(\theta / 2 H_{j}\right)^{p^{\prime}}\left(C E_{2} M_{r} n_{d}^{p^{\prime}}\right)^{p^{\prime}+1}\left(E_{3} M_{r} n_{d}^{p^{\prime}}\right)^{-p^{\prime}} \\
& \quad=\left\{\eta\left(p^{\prime}\right)(\theta / 2)^{p^{\prime}}\left(C E_{2}\right)^{p^{\prime}+1} E_{3}^{-p^{\prime}}\right\} M_{r} \\
& \quad \geq\left\{\eta\left(N^{\prime}\right)(\theta / 2)^{N^{\prime}}\left(C E_{2}\right)^{N^{\prime}+1} E_{3}^{-N^{\prime}}\right\} M_{r}\left(=3 E_{4} M_{r}, \text { say }\right) .
\end{aligned}
$$

If $\left|F\left(x+i t_{m}\right)\right| \leq 2 E_{4} M_{r}$, we put $\varepsilon_{x}=\varepsilon$. Then $0<\varepsilon_{x} \leq \theta / 2 H_{j} \leq 1 / n_{\mu}$ and $|F(\zeta)| \geq E_{4} M_{r}\left(\zeta \in S\left(x+i t_{m}, \varepsilon_{x}\right)\right)$. If $\left|F\left(x+i t_{m}\right)\right| \geq 2 E_{4} M_{r}$, we choose $0<\varepsilon_{x}$ $\leq 1 / n_{\mu}$ so that $|F(\zeta)| \geq E_{4} M_{r}\left(\zeta \in S\left(x+i t_{m}, \varepsilon_{x}\right)\right)$.

Thus we have chosen, for every $x \in\left[r-\theta / H_{0}, r-\theta / H_{\sigma-1}\right]$, a number $0<\varepsilon_{x} \leq 1 / n_{\mu}$ such that $|F(\zeta)| \geq \min \left\{E_{1}, E_{4}\right\} M_{r}\left(\zeta \in S\left(x+i t_{m}, \varepsilon_{x}\right)\right)$.

If $x \in\left[r-\theta / H_{\sigma-1}, r-\theta / n_{\nu}\right]$, we can use the method given in (b), since $\left|c_{\nu}\right| \exp \left(n_{\nu} x\right) \geq M_{r} \exp \left(-\theta n_{\nu} / H_{\sigma-1}\right) \geq \exp \left(-\theta q^{4 \lambda \lambda}\right) M_{r}$. Analogously, we can use the method for $x \in\left[r-\theta / n_{\mu}, r-\theta / H_{0}\right]$. Consequently, in the case $\sigma \geq 2$, we can choose, for every $x \in\left[\alpha_{r}, \beta_{r}\right]$, a number $0<\varepsilon_{x} \leq 1 / n_{\mu}$ satisfying the required inequality with some constant.

In the case $\sigma=0,1$ also, we can use the method given in (b). Thus 
in any case, we can choose, for every $x \in\left[\alpha_{r}, \beta_{r}\right]$, a number $0<\varepsilon_{x} \leq 1 / n_{\mu}$ such that $|F(\zeta)| \geq E M_{r}\left(\zeta \in S\left(x+i t_{m}, \varepsilon_{x}\right)\right)$ ( $E$ : some constant).

Now we choose a finite covering $D\left(x_{j}+i t_{m}, \varepsilon_{x_{j}}\right)(j=1, \cdots, u)$ of $I\left(t_{m}, r\right)$ and put $\Gamma_{m}=\partial\left\{\bigcup_{j=1}^{u} D\left(x_{j}+i t_{m}, \varepsilon_{x_{j}}\right)\right\}$. Then we have $\operatorname{diam}\left(\Gamma_{m}\right) \leq 3 / n_{\mu}=$ $3 / n_{\nu_{m-1}}$, according to diam $\left(I\left(t_{m}, r\right)\right)=\theta / n_{\mu}-\theta / n_{\nu} \leq 1 / n_{\mu}$ and $0<\varepsilon_{x_{j}} \leq 1 / n_{\mu}$ $(j=1, \cdots, u)$. We have also $|F(\zeta)| \geq E M_{r}\left(\zeta \in \Gamma_{m}\right)$. This completes the proof of (B).

\section{§6. Application}

Application 8. In [2], K. G. Binmore showed that an Hadamard lacunary series $f(z)$ given by (1) has no finite asymptotic value if $\limsup _{k \rightarrow \infty}\left|c_{k}\right|>0$. We note that the discussion in the proof of (A) (; in particular (13),) gives a new proof of this fact. For the sake of simplicity, we work only with $\limsup _{k \rightarrow \infty}\left|c_{k}\right|=\infty$.

Let $\tilde{\gamma}$ be a path in $D$ with $b(\tilde{\gamma}) \neq \emptyset$. Without loss of generality, we may assume $b(\tilde{\gamma}) \ni 1$. Then there exists a path $\tilde{\Gamma}$ in $U$ with $b(\tilde{\Gamma}) \ni 0$ and $\iota(\tilde{\Gamma})=\tilde{\gamma}$, where $\iota$ is the mapping defined by $\iota(\zeta)=e^{\zeta}$. It is sufficient to prove that $F(\zeta)$ has no finite asymptotic value along with $\tilde{\Gamma}$. Let $\left(w_{m}\right)_{m=1}^{\infty}$ be a sequence in $\tilde{\Gamma}$ with $\operatorname{Re} w_{m}=\beta_{r_{m}}(m \geq 1)$. Then (13) shows that

$$
\left|F(\zeta)-F\left(w_{m}\right)\right| \geq 3 D M_{r_{m}} \quad\left(\zeta \in S\left(w_{m}, \varepsilon_{w_{m}}\right)\right) .
$$

Let $w_{m}^{\prime}$ be a point in $\tilde{\Gamma} \cap S\left(w_{m}, \varepsilon_{w_{m}}\right)(m \geq 1)$. Then (22) holds for $\zeta=w_{m}^{\prime}$. Since $\lim _{m \rightarrow \infty} M_{r_{m}}=\infty, F(\zeta)$ has no finite asymptotic value along with $\tilde{\Gamma}$.

Application 9. We say that an analytic function $g(z)$ in $D$ is annular if there exists a sequence $\left(\gamma_{m}^{*}\right)_{m=1}^{\infty}$ of Jordan curves in $D$ such that $\left\langle\gamma_{m}^{*}\right\rangle \ni 0$ $(m \geq 1)$ and $\lim _{m \rightarrow \infty} \min \left\{|g(z)| ; z \in \gamma_{m}^{*}\right\}=\infty$. We say that $g(z)$ is strongly annular if we can choose $\left(\gamma_{m}^{*}\right)_{m=1}^{\infty}$ so that $\gamma_{m}^{*}$ 's are circles with center 0 in addition to the above conditions. L. R. Sons showed that an Hadamard lacunary series $f(z)$ given by (1) is annular if and only if $\limsup _{k \rightarrow \infty}\left|c_{k}\right|$ $=\infty$. The "only if" part is immediately seen; if $\limsup _{k \rightarrow \infty}\left|c_{k}\right|<\infty$, then $f(z)$ is normal ([8]) and hence $f(z)$ is not annular ([9] p. 267). Let us show that the "if" part is deduced from $(A)$. Put $I_{m}=\left\{\zeta\right.$; Re $\zeta=\beta_{r_{m}}, 0 \leq \operatorname{Im} \zeta$ $\leq 2 \pi\}(m \geq 1)$. Given $m \geq 1$, (A) shows that, for every $w \in I_{m}$, there exists $0<\varepsilon_{w} \leq 1 / n_{\nu_{m}}$ such that $|F(\zeta)| \geq D M_{r_{m}}\left(\zeta \in S\left(w, \varepsilon_{w}\right)\right)$. We choose a finite covering $D\left(w_{j}, \varepsilon_{w_{j}}\right)(j=1, \cdots, u)$ of $I_{m}$ and put $V_{m}=\iota\left(\bigcup_{j=1}^{u} D\left(w_{j}, \varepsilon_{w_{j}}\right)\right)$. Then $V_{m} \supset S\left(0, \beta_{r_{m}}\right)$. Let $\left(\gamma_{m}^{*}\right)_{m=1}^{\infty}$ be the sequence defined by $\gamma_{m}^{*}=\partial V_{m} \cap$ 
$D\left(0, \beta_{r_{m}}\right)$. Then $\left\langle\gamma_{m}^{*}\right\rangle \ni 0(m \geq 1)$ and $\lim _{m \rightarrow \infty} \min \left\{|f(z)| ; z \in \gamma_{m}^{*}\right\}=\infty$. Hence $f(z)$ is annular.

Let us remark that, in Sons's result, "annular" cannot be replaced by "strongly annular". This is a consequence of the following proposition: Let $\phi(z)=\sum_{k=1}^{\infty} b_{k} z^{\lambda_{k}}$ be an analytic function in $D$ such that, with $s_{m}=$ $\left(\sum_{k=1}^{m}\left|b_{k}\right|^{2}\right)^{1 / 2}(m \geq 1), \lim _{m \rightarrow \infty} b_{m} / s_{m}=0$ and $\liminf _{k \rightarrow \infty} \log \lambda_{k+1} / \log \lambda_{k}>1$. Then $\phi(z)$ is not strongly annular.

The proof is as follows. Nothing is to be proved if $\lim _{m \rightarrow \infty} s_{m}<\infty$. Let $\lim _{m \rightarrow \infty} s_{m}=\infty$. Then the method given in [7] (Lemma 38) yields meas $\left\{t ;\left|\phi\left(\rho e^{i t}\right)\right| \leq 2 \omega\right\} \geq \delta\left(\omega / d_{\rho}\right)^{2} \quad\left(\rho_{0} \leq \rho<1\right)$ for some $0<\rho_{0}<1$, where "meas" signifies the 1-dimensional Lebesgue measure,

$$
\omega=4 \pi \sum_{\ell=2}^{\infty} \sum_{k=1}^{\ell-1}\left|b_{k}\right| \lambda_{k} \mid \lambda_{\ell}, \quad d_{\rho}=\left(\sum_{k=1}^{\infty}\left|b_{k}\right|^{2} \rho^{2 \lambda_{k}}\right)^{1 / 2}
$$

and $\delta$ an absolute constant. Thus $\min \{|\phi(z)| ;|z|=\rho\} \leq 2 \omega\left(\rho_{0} \leq \rho<1\right)$, and hence $\limsup _{\rho \rightarrow 1} \min \{|\phi(z)| ;|z|=\rho\} \leq 2 \omega$. This shows that $\phi(z)$ is not strongly annular.

\section{REFERENCES}

[1] J. M. Anderson, Boundary properties of analytic functions with gap power series, Quart. J. Math. Oxford Ser. (2), 21 (1970), 247-256.

[2] K. G. Binmore, Analytic functions with Hadamard gaps, Bull. London Math. Soc., 1 (1969), 211-217.

[ 3 ] K. G. Binmore-R. Hornblower, Boundary behaviour of functions with Hadamard gaps, Nagoya Math. J., 48 (1972), 173-181.

[ 4 ] W. H. J. Fuchs, On the zeros of power series with Hadamard gaps, Nagoya Math. J., 29 (1967), 167-174.

[5] G. R. Maclane, Asymptotic values of holomorphic functions, Rice Univ. Stud., 49 no. 1 (1963), 3-83.

[6] T. Murai, The value-distribution of lacunary series and a conjecture of Paley, Ann. Inst. Fourier (Grenoble) (1), 31 (1981), 131-152.

[ 7 ] — On lacunary series, Nagoya Math. J., 85 (1982), 87-154.

[ 8 ] Ch. Pommerenke, On Block functions, J. London Math. Soc. (2), 2 (1970), 689-695.

[9] - Univalent functions (Vandenchoeck and Ruprecht 1975).

[10] L. R. Sons-D. M. Champbell, Hadamard gap series and normal functions, Bull. London Math. Soc., 12 (1980), 115-118.

[11] M. Weiss, Concerning a theorem of Paley on lacunary power series, Acta Math., 102 (1959), 225-238.

Department of Mathematics

Faculty of Science

Nagoya University

Chikusa-ku, Nagoya, 464

Japan 